\title{
Laser Photolithography of Graphene Oxide
}

\section{Barbara NASIłOWSKA ${ }^{1,1}$, Magdalena Naurecka ${ }^{2,1}$, Zdzisław BOGDANOWICZ ${ }^{3,2}$, Zygmunt MIERCZYK ${ }^{4,1}$}

\author{
${ }^{1}$ Institute of Optoelectronics, Military University of Technology, Warsaw, Poland \\ ${ }^{2}$ Faculty of Mechanical Engineering, Military University of Technology, Warsaw, Poland \\ E-mails: ${ }^{1}$ barbara.nasilowska@wat.edu.pl (corresponding author), ${ }^{2}$ magdalena.naurecka@wat.edu.pl. ${ }^{3} z d z i s l a w$.

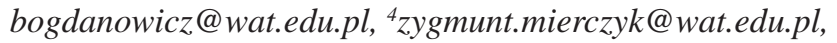

\begin{abstract}
The paper presents the results of research on the effect of laser photolithography on graphene oxide (GO) fixed on the silicon wafer and PVC plate (polyvinyl chloride). Before fixing the graphene oxide, the surface was treated with plasma cleaning in the argon atmosphere. Then deposition with AZ4533 photoresist and laser photolithography were carried out. Analysis of the spectral characteristics and structural tests proved that graphene oxide was not damaged during exposing to a laser beam. The laser photolithography process was followed with observation of typical FTIRATR bonds originating from $\mathrm{COOH}, \mathrm{C}=\mathrm{O}$ and $\mathrm{C}-\mathrm{O}-\mathrm{C}$ typical for $\mathrm{GO}$. The pattern (on the $\mathrm{GO}+\mathrm{AZ} 4533$ coating) was exposed to the laser beam $(\mathrm{GaN})$ and revealed by a developing agent. That was a base for continuation of further research with a purpose of ion etching.
\end{abstract}

KEY WORDS: photolithography, grapheme oxide, wafer silicon, PVC

\section{Introduction}

Graphene oxide (GO) is a potential precursor for graphene [1]. Paper [2] shows how the electron beam lithography (EBL) was used for preparing nanostructured reduced patterns on the GO paper surface, while preserving its mechanical resistance and flexibility. Different EBL parameters, like dose and time of exposure for patterning were tested. SEM analysis showed the consequent increase of contrast of the reduced stripes on the patterned regions due to the increase of electron beam doses [2]. Moreover, surface potential microscopy experiments also exhibited a clear contrast between the patterned and non-patterned regions [2].

Gonçalves and co. [3] showed how by controlling the depth of laser beam focus during laser lithography on a graphene oxide (GO) film, a rGO/GO/rGO structure is formed in the GO film. Subsequently, two independent interdigitated electrodes (IDEs) were fabricated on the top and bottom of the GO film surface.

In $[2,3,5]$ papers present photolithography directly on graphene or graphene oxide, but laser photolithography of graphene oxide coated with photoresist has been not yet examined.

The purpose of our experiments was to verify the possibility of using laser photolithography for patterning of graphene layers for photonics usage.

\section{Methodology}

After performing plasma cleaning in the argon atmosphere (100W) (Fig. 1a), PVC plate and a silicon wafer were placed in the rotary centrifuge, where graphene oxide in the form of suspension was fixed. Then, AZ 4533 photoresist was applied (Fig. 1c) and heated in the POLOS Precision Hotplate.

\footnotetext{
${ }^{1}$ Corresponding author. Tel.: 600270968

E-mail address: barbara.nasilowska@wat.edu.pl
} 
a)

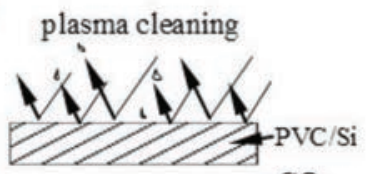

b)

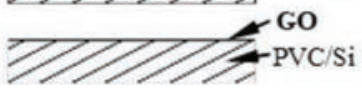

c)

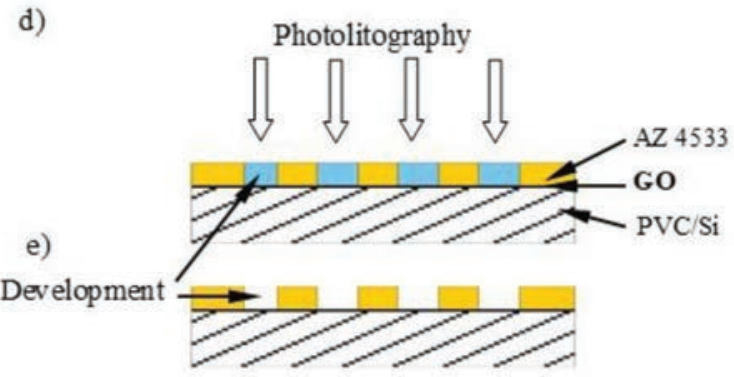

Fig. 1. Execution diagram; plasma clearing of the surface: (a), fixing with graphene oxide (b), covering with photoresist (c), laser photolithography (d), developing of the exposed pattern (e).

For exposing the pattern with a semi-conductor laser GaN (Fig. 2) in the photolithography process, laser power within the range of $180 \div 370 \mathrm{~mJ} / \mathrm{cm} 2$ was applied (Fig. 1d). At the last stage, the exposed pattern was developed. The surface was subjected to FTIR-ATR spectrometer (Fig. 2b) and for structural analysis with an electron scanning microscope (Fig. 2c).
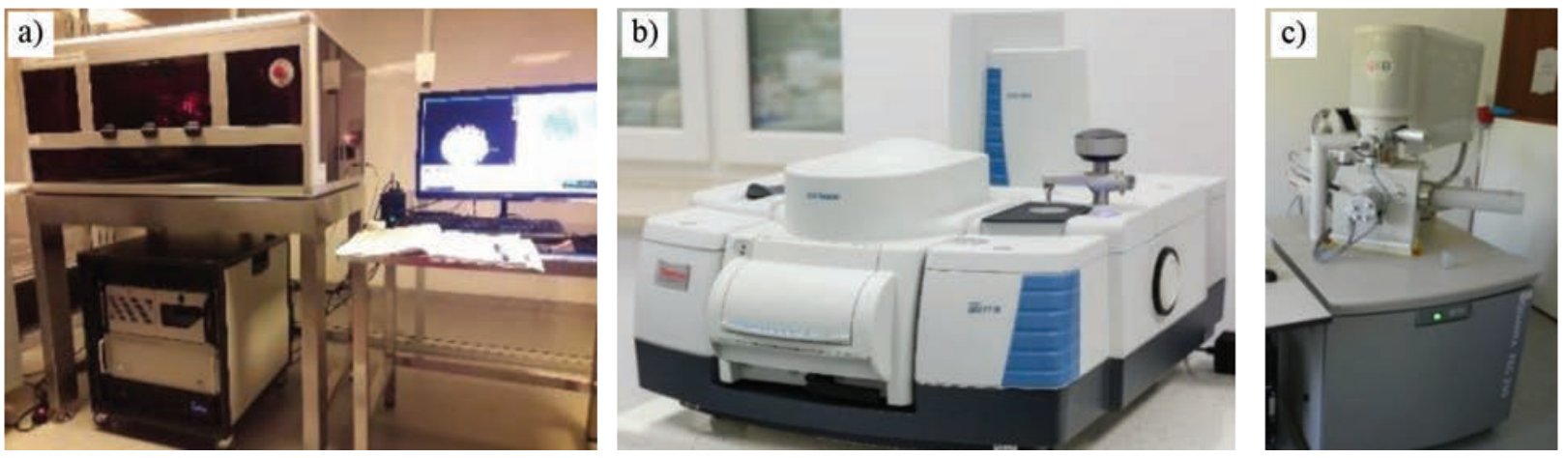

Fig. 2. The LaserWriter system: (a), Thermo Fisher Scientific FTIR iS50 spectrometer (b), Quanta 250 FEG electron scanning microscope (c).

\section{Results and Discussion}

Analysis of the structural tests also proved that graphene oxide did not undergo any change after applying, centrifuging and heating of the photoresist (ZA4533). The surface layer of the graphene oxide and photoresist applied on the silicon wafer and PVC card was characterized by homogeneity (Fig. 3).
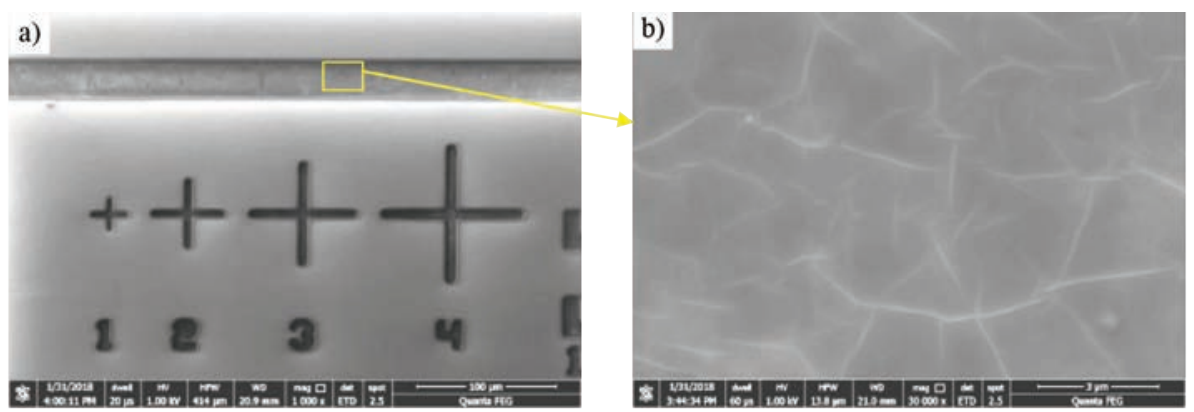

Fig. 3. SEM image of graphene oxide surface before and after laser photolithography. 
Before and after laser photolithography, within the spectrum range of $1200 \div 1700 \mathrm{~cm}^{-1}$ the bands typical for $\mathrm{GO}$ originating from $\mathrm{COOH}, \mathrm{C}=\mathrm{O}$ and $\mathrm{C}-\mathrm{O}-\mathrm{C}$ were observed $[4,6]$. Structural tests and analysis of the photolithography spectrum did not affect the quality of the graphene oxide surface (Fig. 4).

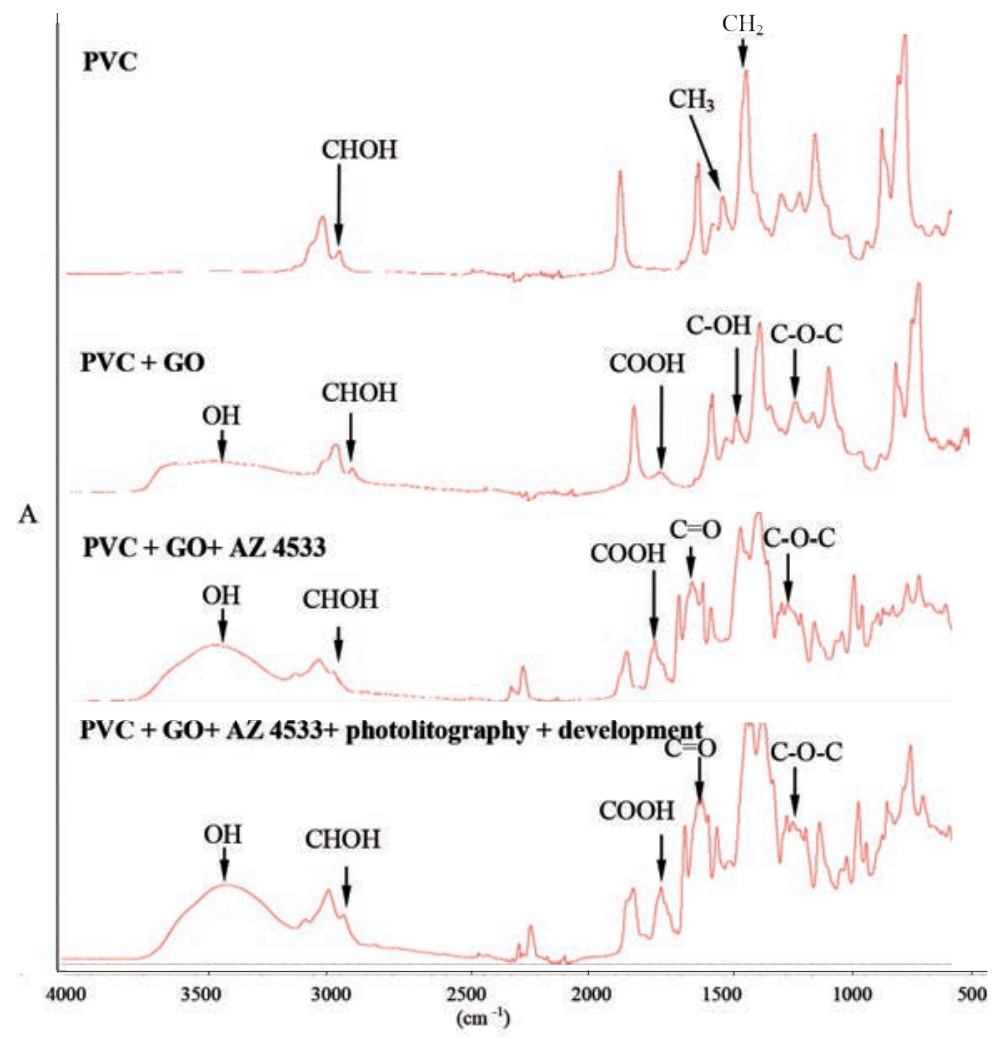

Fig. 4. FTIR-ATR spectrum; PVC, PCV + graphene oxide (GO), PCV + graphene oxide (GO) + ZA4533 photoresist, $\mathrm{PCV}+$ graphene oxide $(\mathrm{GO})+\mathrm{AZ} 4533$ photoresist after laser photolithography and developer.

\section{Conclusion}

Graphene oxide was not damaged due to interaction of the GaN laser beam $\left(180 \div 370 \mathrm{~mJ} / \mathrm{cm}^{2}\right)$. While applying the proper preparatory procedures (centrifuging and heating) of the AZ4533 photoresist, a photosensitive coating sufficiently adsorbs graphene oxide (GO). However, covering the PVC plates and silicon wafer with graphene oxide with a rotational speed of centrifuging $3500 \div 4000$ rotations $/ \mathrm{min}$ needs to be considered as too low. Such speed accumulates air bubbles in the surface layer which prevents homogeneous covering with photoresist.

\section{Acknowledgemen}

This work was by a Grant-in-Aid for projects ZBW/08-895/2020/WAT.

\section{References}

1. Pfaffeneder-Kmen M., Falcon Casas I., Naghilou A., Trettenhahn G., Kautek W., Electrochimica Acta, 255, 2017, p. 160-167.

2. Okamura H., Kayanoki M., Takada K., Nakajiri H., Muramatsu K., Yamashita M., Shirai M., Polym. Adv. Technol., 23, 2012, p. 1151.

3. Gonçalves G., Borme J., Bdkin I., Mayorga A. G., Irurueta G., Nogueira H. I.S., Serrano M. C., Alpuim P., P. A.A.P. Marques, Carbon 129, 2018, p. 63-75.

4. Soongeun K., Daekyeong J., Hyungjun L., Geehong K., Kee-Bong C., JaeJong L., Applied physics letters III, 143903, 2017, p. 63-75.

5. Guo W., Chen J., Sun S., Zhou Q., J. Phys. Chem. C, 120, 2016, p. 7451-7456.

6. Hondred J. A., Stromberg L. R., Moshe C. L., Claussen J. C., ACS Nano, 11, 2017, p. 9836-9845.

7. Changa X., Wanga Z., Quana S., Xua Y., Jianga Z., Shaoa L., Applied Surface Science, 316, 2014, p. $537-548$. 